\title{
Sexual conflict over the duration of copulation in Drosophila montana: why is longer better? Dominique Mazzi*†1,2, Jenni Kesäniemi ${ }^{1}$, Anneli Hoikkala ${ }^{1}$ and Kirsten Klappert*†3,4
}

Address: ${ }^{1}$ Department of Biological and Environmental Science, University of Jyväskylä, PO Box 35, FIN-40014, Finland, ${ }^{2}$ ETH Zurich, Institute of Plant Sciences, Applied Entomology, Schmelzbergstrasse 9, CH-8092 Zurich, Switzerland, ${ }^{3}$ Evolutionary Biology, Dyer's Brae House, University of St Andrews, St Andrews, Fife, KY16 9TH, UK and ${ }^{4}$ Aquatic Ecology, EAWAG/ETH, Ueberlandstrasse 133, CH-8600 Duebendorf, Switzerland

Email: Dominique Mazzi* - dominique.mazzi@ipw.agrl.ethz.ch; Jenni Kesäniemi - jeemkesa@cc.jyu.fi; Anneli Hoikkala - anhoikka@bytl.jyu.fi; Kirsten Klappert* - Kirsten.Klappert@eawag.ch

* Corresponding authors †Equal contributors

Published: 12 June 2009

BMC Evolutionary Biology 2009, 9:132 doi:10.1/86/147|-2/48-9-132

This article is available from: http://www.biomedcentral.com//47|-2/48/9//32

(c) 2009 Mazzi et al; licensee BioMed Central Ltd.

This is an Open Access article distributed under the terms of the Creative Commons Attribution License (http://creativecommons.org/licenses/by/2.0), which permits unrestricted use, distribution, and reproduction in any medium, provided the original work is properly cited.
Received: 12 December 2008

Accepted: 12 June 2009

\begin{abstract}
Background: Conflicts of interest between the sexes are increasingly recognized as an engine driving the (co-)evolution of reproductive traits. The reproductive behaviour of Drosophila montana suggests the occurrence of sexual conflict over the duration of copulation. During the last stages of copulation, females vigorously attempt to dislodge the mounting male, while males struggle to maintain genital contact and often successfully extend copulations far beyond the females' preferred duration.
\end{abstract}

Results: By preventing female resistance, we show that females make a substantial contribution towards shortening copulations. We staged matings under different sex ratio conditions, and provide evidence that copulation duration is a form of male reproductive investment that responds to the perceived intensity of sperm competition as predicted by game theoretical models. Further, we investigated potential benefits to persistent males, and costs to females coerced into longer matings. While males did not benefit in terms of increased progeny production by protracting copulation, female remating was delayed after long first copulations.

Conclusion: Copulation time is a trait subject to sexual conflict. Mating durations exceeding female optima serve males as a form of 'extended mate guarding': by inducing mating refractoriness in the female, a male extends the time over which its sperm is exclusively used to sire progeny and reduces the likelihood of the female being reinseminated by a competitor.

\section{Background}

While sexual selection has long been regarded as the engine responsible for the co-evolution of male traits and female preferences [1], antagonistic co-evolution between the sexes has only recently gained growing attention (reviewed in [2]). Evolutionary conflicts of interest between the sexes are common, and increasingly recog- nized as a powerful force driving the evolution of traits involved in reproduction [3-6]. Sexual conflict is expected to fuel the evolution of adaptations that bias the outcome of reproduction towards one sex, even at the expense of the fitness interests of the other. In response, the latter sex should evolve countermeasures that effectively minimize the costs of sexual interactions. Heightened resistance in 
turn promotes further persistence, leading to an escalating co-evolutionary arms race similar to the Red Queen dynamics in parasite-host relationships [7]. The intraspecific conflict between males and females arises for control over aspects of reproduction such as mating rate, female propensity to remate, female proximate fecundity, or relative parental effort (reviewed in [4]).

The optimal outcome of reproductive interactions for males and females rarely, if ever, coincides [8]. In promiscuous species where sperm competition occurs, males can enhance their reproductive success by either increasing the number of inseminated partners or by preventing their partners from remating. The two strategies are to some extent mutually exclusive, since the time and energy budgeted on a given mating will be traded against the acquisition of further matings [9]. Hence, whenever males incur nontrivial mating costs, they should prudently partition reproductive effort over a series of matings to maximize lifetime reproductive success (reviewed in [10]). The operational sex ratio (OSR, the ratio of males to females ready to mate, [11]) is a reliable predictor of the probability that a male will have to outcompete sperm of rival males to fertilize a female's eggs. While reproductive investment is predicted to rise linearly with the degree of sperm competition across species and indeed does so in several groups (e.g. [12-16]), a different pattern is predicted within species. Within species, a male's expenditure should not only depend on the risk of sperm competition (i.e. competitors present or not) but also on the intensity of competition (i.e. the number of competitors). Parker et al's [17] series of evolutionarily stable strategy models predicts that males should invest little when there are no competitors and most in the presence of one competitor. Somewhat counter intuitively, as the number of competitors exceeds two the models predict a corresponding decrease in expenditure because the marginal gains from any additional unit of investment decrease. Empirical tests have provided some, albeit not ubiquitous, support for a curvilinear relationship between competition intensity and male investment ([18,19] but see e.g. [20]). When facing competition in fertilising eggs, males of some species have been found to prolong copulation duration, e.g. [21].

Long-lasting copulations are widespread in insect species, despite associated potential costs in terms of time, energy, increased predation vulnerability and disease transmission $[15,22]$. Two main hypotheses, 'the ejaculate transfer hypothesis' and 'the extended mate guarding hypothesis', have been put forward to explain the advantage of protracted mating associations. The 'ejaculate transfer hypothesis' presupposes a more or less linear relationship between copulation duration and sperm transfer and storage, and suggests that longer copulations facilitate ejaculate transfer and lead to increased female lifetime fecundity (see e.g. [23]). In several species, sperm is known to be transferred only at the very beginning of copulation, while during the later stages of copulation males transfer substances that build effective mating plugs or other manipulating seminal fluids $[24,25]$. The 'extended mate guarding hypothesis' posits that with longer mating, the chances of the female being inseminated by a subsequent male are reduced $[9,22,26]$. Prolonged copulation durations have been found to act directly as mate guarding (reviewed in [26]). To understand the adaptive significance of prolonged copulations, it is necessary to identify which sex controls the duration of copulation. Among Drosophila species, copulation duration has been found to be largely under male control in $D$. melanogaster, $D$. simulans, D. mojavensis and D. athabasca [27-31] or determined through interactions between males and females in D. elegans [32]. A recent study of the functional significance of external genital structures in D. melanogaster and D. simulans [33] confirmed that females are coerced into mating for as long as suits the males' interests. Here, the male control relies on a 'lock'-like genital coupling mechanism and females are unable to break the lock without sustaining harm. While there is ample evidence for anatomical modifications enabling males to coerce females into mating, so far no indication has been found in Drosophilidae of modifications of the female genitalia that may have evolved to defy male control, as documented in other insects [34]. In general, up to date, reports of (at most partial) female control over copulation duration are sparse (e.g. in D. mojavensis [30]). The ejaculate transferred to females consists not only of sperm but also seminal fluids. In several species, seminal fluids have been shown to contain substances that manipulate female physiology and behaviour [35]. Possible manipulations e.g. in D. melanogaster [36] include prolonged latency to remating or preventing remating altogether, decrease of subsequent copulation durations, enhanced reproductive output, sometimes at the females' expense by derogating longevity, alteration of immune response and feeding behaviour, increased storage and utilization of sperm.

Accessory gland proteins (acps) most likely evolved to stimulate reproductive processes in order to synchronise female receptivity with male reproductive effort. However, these substances can be deleterious in high doses [37] and males can utilize them to manipulate female physiology and behaviour to their advantage. Seminal fluid proteins evolve much faster than non-reproductive proteins, providing indirect evidence for sexual selection or an underlying arms race of sexual antagonistic co-evolution between male coercion and female resistance (see [4] for a review on acps and sexual conflict in Drosophila).

In Drosophila montana, a promiscuous species in the large Drosophila virilis species group, males and females apparently undergo a conflict of interest during copulation. Once a female has allowed a courting male to mount, and 
after an initial phase of apparent harmony, copulating pairs enter an obvious conflict phase, where females conspicuously attempt to dislodge the mounting male by vigorously kicking with their hind legs, wing flicking or decamping. While there is good knowledge of sexual selection processes in D. montana and other species of the D. virilis species group (reviewed in [38]), little is known about the potential for sexually antagonistic co-evolution in this group. Our aim was to explain the males' efforts to prolong matings beyond the females' preferred duration. First, we tested whether copulation duration is a form of reproductive investment that responds to variation in the OSR as predicted by sperm competition game models. Second, we investigated which of the sexes exerts control over the duration of copulation by comparing the duration of copulation of unmanipulated females and of females whose resistance attempts were precluded. Third, we addressed potential costs arising from prolonged copulations to females and potential benefits accruing to perseverant males, in terms of reproductive output and in terms of the share of paternity in the face of competition against a later rival.

\section{Results \\ Sex ratio manipulation}

Across matings, the durations of the struggle phase and of the entire copulation were significantly positively correlated $(\mathrm{N}=115, \mathrm{r}=0.56, \mathrm{P}<0.001)$, indicating that perseverant males indeed obtained increased mating time. The length of copulation until the onset of female reluctance and the length of the struggle were significantly negatively correlated $(\mathrm{N}=115, \mathrm{r}=-0.22, \mathrm{P}=0.017)$. Hence, the earlier on males were rejected, the longer they struggled to hold on. Conversely, males that had been tolerated for longer already soon gave up when rejected, or even disengaged on their own accord.

In agreement with theoretical predictions, matings were longest in the presence of two males contending for a single female, shortest when five males were competing, and intermediate at even sex ratios (Figure 1, bars to the right of the dashed line). Even though conventional (nondirectional) analysis of variance failed to reveal significant effects of the sex ratio manipulation, the means of copulation duration precisely matched our a priori ordered hypothesis (one-way ANOVA, $\mathrm{F}_{3,111}=2.09 ; \mathrm{OH}$-test, $\mathrm{r}_{\mathrm{s}} \mathrm{P}_{\mathrm{c}}=$ $\left.0.85, \mathrm{P}_{\mathrm{dir}}<0.01\right)$. Alternative hypotheses linking the duration of matings and struggles with the total density of individuals, or with the density of one sex (e.g. because of changes in the availability of potential mates or in the occurrence of intrusions) are not supported by the observed response pattern.

\section{Control over copulation duration}

When females were prevented from dislodging the copulating male, matings lasted one and a half times longer than when female movement was unconstrained (mean \pm SD $377 \pm 90 \mathrm{~s}$ versus $248 \pm 38 \mathrm{~s}, \mathrm{t}=6.58$, d.f. $=39, \mathrm{P}<$ 0.001 , two leftmost bars in Figure 1). Hence, female reluctance behaviour effectively contributes to reducing copulation durations.

\section{Mating interruptions}

The reproductive output in terms of egg production, egg hatching success and larvae production of females whose matings were interrupted at predetermined times showed an overall positive relationship with copulation duration, up to a certain plateau (Figures $2 \mathrm{a}-\mathrm{c}$ ). None of the matings interrupted within one minute of initiation produced any offspring. Female productivity rose steeply during the second minute into copulation but soon levelled off, such that not significantly fewer eggs or progeny were produced in matings interrupted two minutes after their commencement than in naturally terminated matings (mean \pm SE of naturally terminated copulations combined from egg count and larvae count $193.5 \pm 5.1 \mathrm{~s}$ )

Within groups of females that were allowed to terminate matings naturally, progeny production bore no relationship to copulation duration (linear and quadratic fits, all $\mathrm{R}^{2}<0.07$, all $\mathrm{P}>0.3$ ). One out of 26 unmanipulated matings in the egg production and hatching success assay, and two out of 18 unmanipulated matings in the larvae production assay yielded no eggs, giving an estimate of female infertility rate of ca 4 to $11 \%$. For comparison, ca $3 \%$ of the females whose mating was interrupted (one after $60 \mathrm{~s}$ and two after $90 \mathrm{~s}$, out of 114 total) did not lay any eggs (calculated from the egg production assay sample). Three of the 26 unmanipulated matings that did yield eggs did not result in viable offspring, providing an estimate of male sterility of ca $12 \%$, possibly inflated by environmental causes of egg mortality.

The dynamics of sperm transfer closely matched the pattern inferred from the female productivity data. No sperm was recovered from females whose matings lasted less than one minute. The proportion of inseminated females asymptotically increased in subsequent intervals to reach a plateau at around $150 \mathrm{~s}$, after which time $90 \%$ to $100 \%$ of all females were inseminated (non-linear logistic regression on ln-transformed copulation durations, Wald $=17.6, \mathrm{P}<0.001$, with $80 \%$ correctly classified cases, Figure 3). Accordingly, inseminated females experienced significantly longer copulations than non-inseminated ones (Wilcoxon rank sum test, $\mathrm{N}_{\text {inseminated }}=26, \mathrm{~N}_{\text {non-inseminated }}=$ 29, $\mathrm{Z}=-4.64, \mathrm{P}<0.001)$.

\section{Double matings \\ Female remating}

All females that were allowed only $30 \mathrm{~s}$ for the first copulation remated in the remating experiment. Then the proportion of remating females dropped in each of the time 


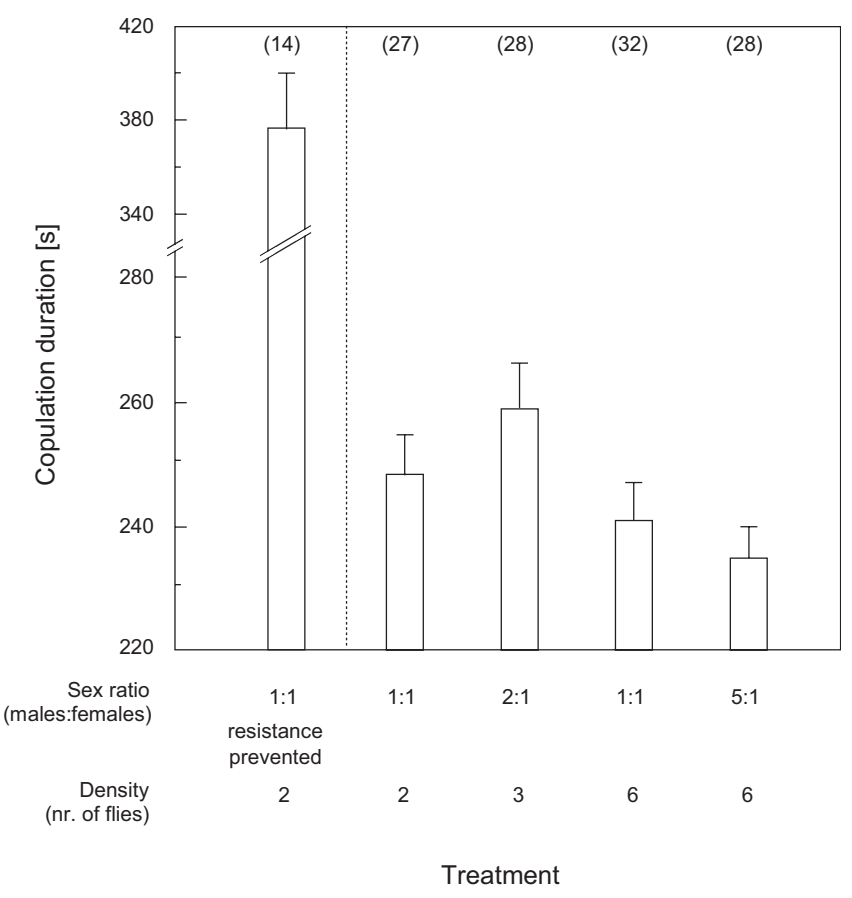

Figure I

Duration of copulations with prevented female resistance and under different sex ratios. The bar to the left of the dotted line shows copulation duration in seconds when females were prevented from dislodging the mounting male (mean $\pm \mathrm{SE}$, note the broken scale on the $y$ axis), bars to the right of the dotted line show duration of copulation (mean $\pm \mathrm{SE}$ ) for different sex ratio manipulation treatments. Sample sizes are given in parentheses.

classes to about one half in the class where the first copulation was interrupted after $120 \mathrm{~s}$. The remating rate remained largely unchanged for first copulations that lasted longer than $120 \mathrm{~s}$ (Figure 4). Accordingly, females that remated had experienced significantly shorter first copulations than females that did not remate (Wilcoxon rank sum test, $\mathrm{N}_{\text {remated }}=109, \mathrm{~N}_{\text {non-remated }}=60, \mathrm{Z}=3.58, \mathrm{P}$ $<0.001)$.

Females that did eventually remate did so faster, the sooner their first copulation had been interrupted (logistic regression, Wald $=11.8, \mathrm{P}=0.001$, with $63 \%$ correctly classified classes, Figure 5). Latency to remating was significantly shorter when the first copulation had been interrupted after $60 \mathrm{~s}$ than when the first copulation was terminated naturally (mean difference $68 \pm 20 \mathrm{~min}, \mathrm{P}=$ $0.016)$ or interrupted after $150 \mathrm{~s}(60 \pm 19 \mathrm{~min}, \mathrm{P}=0.035)$. A trend is still present in the $90 \mathrm{~s}$ class as compared to naturally ended first copulations $(56 \pm 20 \mathrm{~min}, \mathrm{P}=0.099$; overall model ANOVA on square root transformed latencies, $\mathrm{F}_{6,108}=3.42, \mathrm{P}=0.004$, significance of mean differ- ences between groups determined with Tukey HSD posthoc tests).

\section{Paternity analyses}

In remating experiments where the first mating was interrupted while the second was allowed to be completed, the proportion of progeny sired by the first male relative to a subsequent rival $\left(\mathrm{P}_{1}\right.$, a defensive measure of sperm competition) increased with the duration of the first mating (Figure 6). However, the paternity share of the first male (corrected for the confounding effect of the duration of the second copulation by using the regression residuals for analysis) rose only up to a copulation duration of about $120 \mathrm{~s}$ and remained stable thereafter at the level of naturally terminated matings $\left(\mathrm{ANOVA} \mathrm{F}_{4,36}=2.97, \mathrm{P}=\right.$ 0.034 , Dunett's t posthoc test: naturally terminated control copulations were used as control against which all other groups were tested. $90 \mathrm{~s}$ mean difference in paternity of $\mathrm{P}_{1}$ to naturally terminated control group $-25.3 \%, \mathrm{P}=$ $0.013 ; 120 \mathrm{~s}$ to control $-18.8 \%, \mathrm{P}=0.068)$. Conservatively, the comparison neglects females that did not remate (i.e. strictly, $P_{1}=1$ ), which were often precisely those experiencing the longest copulations. Similarly conservatively, we did not determine $\mathrm{P}_{1}$ for pairs with copulation durations shorter than $90 \mathrm{~s}$, because of previous indications that no sperm is transferred within such a short time frame and thus that the second male to mate would sire all of the produced offspring $\left(P_{1}=0\right)$.

\section{Discussion}

\section{Sex ratio manipulation}

Male D. montana gauged the intensity of competition from rival males and adjusted the duration of copulation, used as a proxy of reproductive investment, accordingly. Copulations were longest in the presence of one potential rival, shortest in the presence of four potential rivals, and intermediate in the absence of competition. The pattern thus precisely matches theoretical predictions based on diminishing returns per unit of expenditure with increasing competition [17]. Parker et al.'s [17] evolutionary stable strategy (ESS) models were originally derived to determine optimal sperm expenditure in external spawners, but they have been equally applied to internally fertilizing species in which ejaculates compete in a fair raffle, i.e. in which fertilization success is proportional to the number of sperm transferred [20,39]. When the raffle is loaded, i.e. when one male's sperm is devalued relative to the sperm of another male, the ESS depends on the information the parties have about their role, and whether or not their roles are occupied randomly [40]. In D. montana, the raffle is loaded in favour of the last male (see Results, also [41]). All our experimental males housed for the time of the experiment with one or more rivals mated under the overt threat of not being the last male to mate. While 
a)

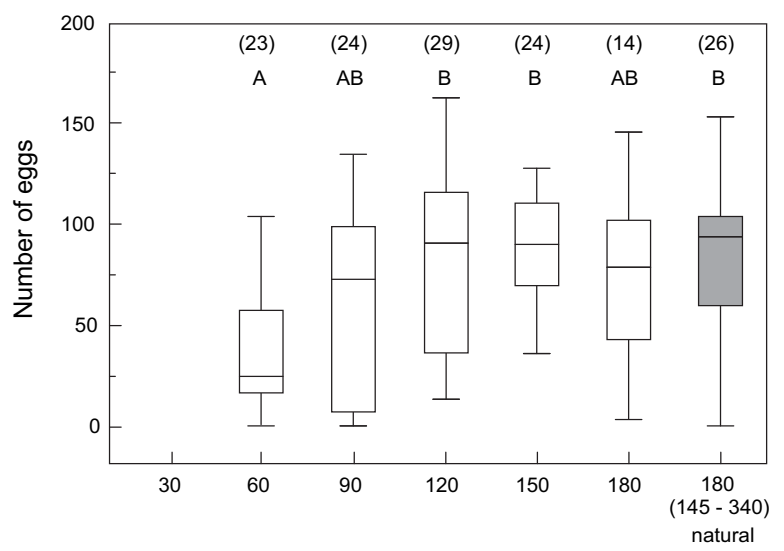

b)

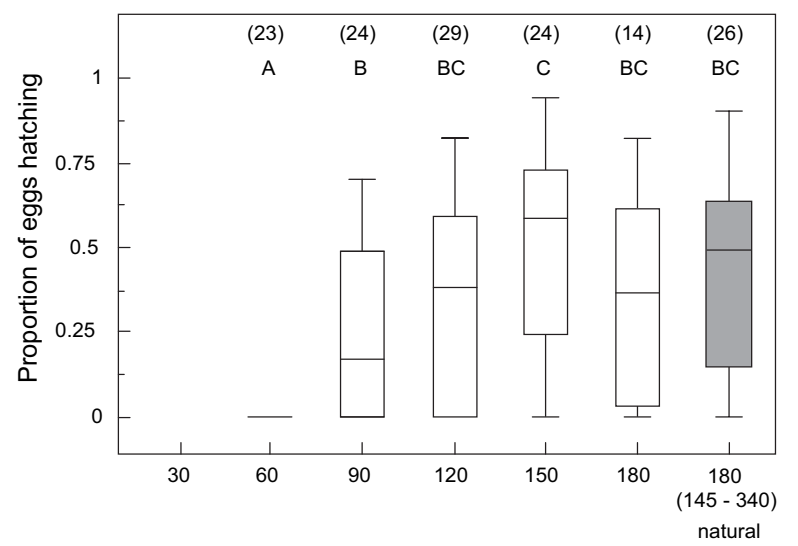

c)

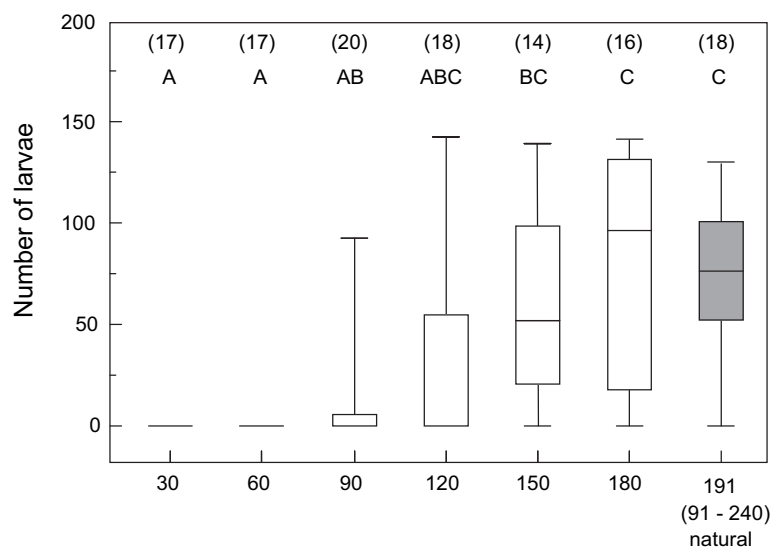

Duration of copulation [s]

Figure 2

Female reproductive output depending on copulation duration. Female reproductive output in terms of (a) egg production, (b) egg hatching success, and (c) larvae production for matings interrupted at predetermined times (white boxes) and naturally terminated matings (shaded box). The box plots represent the median as the middle line, 25 and $75 \%$ quantiles as box boundaries, and 10 and $90 \%$ quantiles as whiskers. The median (range) duration of copulation for unmanipulated matings is given under the corresponding box. Classes not sharing the same letter are significantly different (all $\mathrm{H}>23.6$, all $\mathrm{P}<0.0 \mathrm{I}$, from a Kruskal-Wallis ANOVA followed by Tukey-type multiple comparisons). Sample sizes are given in parentheses. 


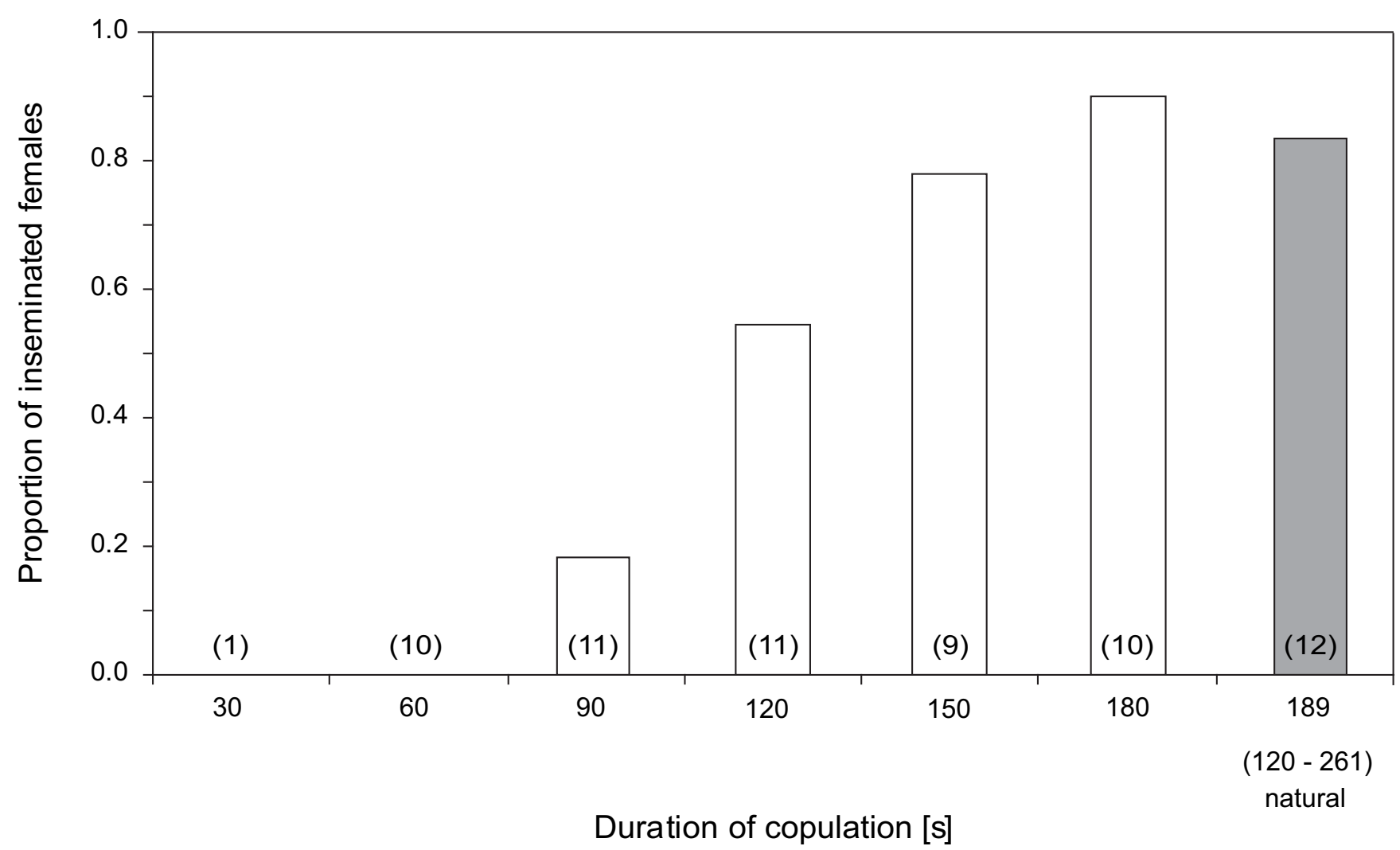

Figure 3

Proportion of inseminated females depending on copulation duration. Proportion of inseminated females for matings interrupted at predetermined times (white bars) and naturally terminated matings (shaded bar). The median (range) duration of copulation for unmanipulated matings is given under the corresponding bar. Sample sizes are given in parentheses.

we used only virgin females in our study, in nature most matings will involve non-virgins, and most males will not be their partner's last mate. However, copulation durations in our double mating experiments suggest that the ability of $D$. montana males to distinguish virgin from mated females is at most imperfect.

A few studies have addressed the question of how flexible the response to changes in OSR (operational sex ratio) is. OSR can fluctuate substantially in both space and time [42], and the individual ability to promptly track changes in OSR would optimize the adjustment of investment to the prevailing circumstances [43-45]. For D. montana males used in our sex ratio manipulation, the effect of ambient OSR overshadowed the effect of the premating OSR (all males having spent practically their entire adult life in single-sex groups). Species of the $D$. virilis group have a short reproductive season in spring, during which flies of both sexes converge on food patches of rotting plant material to mate $[46,47]$. Under such a 'sex at the restaurant' scenario, it makes adaptive sense to respond to the current OSR, rather than to the OSR experienced beforehand elsewhere.

\section{Control over copulation duration}

In contrast to earlier reports that copulation duration is ultimately under the control of males in various Drosophila species [27-31], we showed that D. montana females make a substantial contribution towards shortening the duration of copulation. When female resistance attempts were suppressed, males persisted in copula far longer than they ever managed to in unmanipulated matings. To a human observer, the females' desperate efforts to shake off the mounted male appear to reflect a premium for keeping matings within a certain optimal time frame or, conversely, a cost from matings that last longer than required from their own perspective.

\section{Costs and benefits of prolonged copulation durations}

We explicitly addressed and documented a potential cost accruing to females coerced into exceedingly long matings, relative to their preferred duration. The longer the females' first copulation, the lower the proportion of females that remated on the following day, and the longer the remating latency for eventually remating females. Males clearly profit from a delay in subsequent female remating through the extended period during which their 


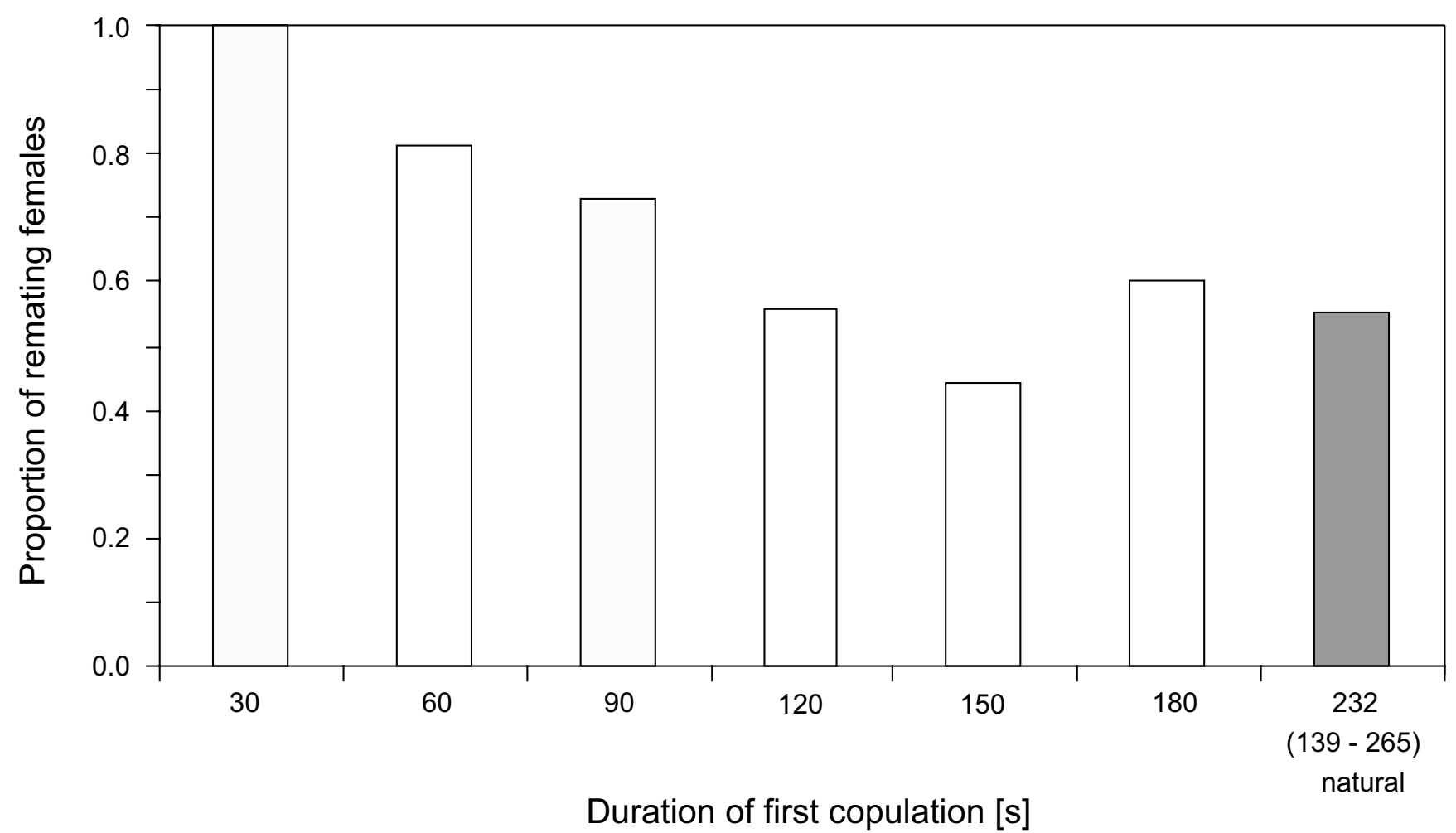

Figure 4

Proportion of females that remate depending on duration of first copulation. Proportion of remating females when first matings had been interrupted at predetermined times (white bars) or allowed to be terminated naturally (shaded bar). The median (range) duration of copulation for unmanipulated first matings is given under the corresponding bar.

sperm is used exclusively to sire progeny (see also [48]). Nearly all females were inseminated well before the end of naturally terminated copulations. Thus, the 'ejaculate transfer hypothesis', which postulates that copulation duration and sperm transfer/storage are positively correlated, does not fit the observed pattern as well as the 'extended mate guarding hypothesis'. The latter hypothesis provides here a better explanation, as the prolonged remating latency in females after longer copulations reduces the likelihood of females being inseminated by a subsequent male. The onset of female reluctance behaviour after sperm transfer seems to have reached its maximum suggests that males avail themselves of proteins in the seminal fluid to modify female behaviour to their advantage, as has earlier been shown in other species $[36,49]$. Also, the fact that females produced eggs after copulations lasting less than required for sperm transfer to occur (90 s) might be attributed to the action of fecundityenhancing seminal fluids transferred at the very beginning of copulation, even before sperm transfer begins. Even though an induced refractoriness can under certain circumstances be beneficial to females [50], it turns detrimental when it drives female mating rate away from the optimum, and obviously compromises female interests when it curtails the benefits to be derived from multiple mating.

Arnqvist and Nilsson [50] provide convincing arguments for the claim that a single mating does not in general maximize lifetime fitness of female insects. Even in species without nuptial feeding, direct benefits of remating in the form of replenished sperm supplies, stimulating or gonadotropic effects of mating, and nutritional or hydrating effects of male ejaculate substances more than offset negative effects for moderate remating rates. Another direct benefit of multiple matings for females lays in counteracting male sterility, which can be as substantial as the approximate $12 \%$ we estimated in male D. montana. Additional indirect benefits accruing to females from mating with different partners (i.e. polyandry) such as increased offspring diversity and the opportunity for bet-hedging against genetically incompatible or inferior males $[51,52]$ further promote and maintain female remating behaviour. Aspi's [53] survey of a natural population of D. montana in Finland revealed that virtually all sampled females carried sperm of at least two males. In the field, copulations were commonly observed posterior to the date when $95 \%$ of all the females were inseminated [47]. In the lab- 


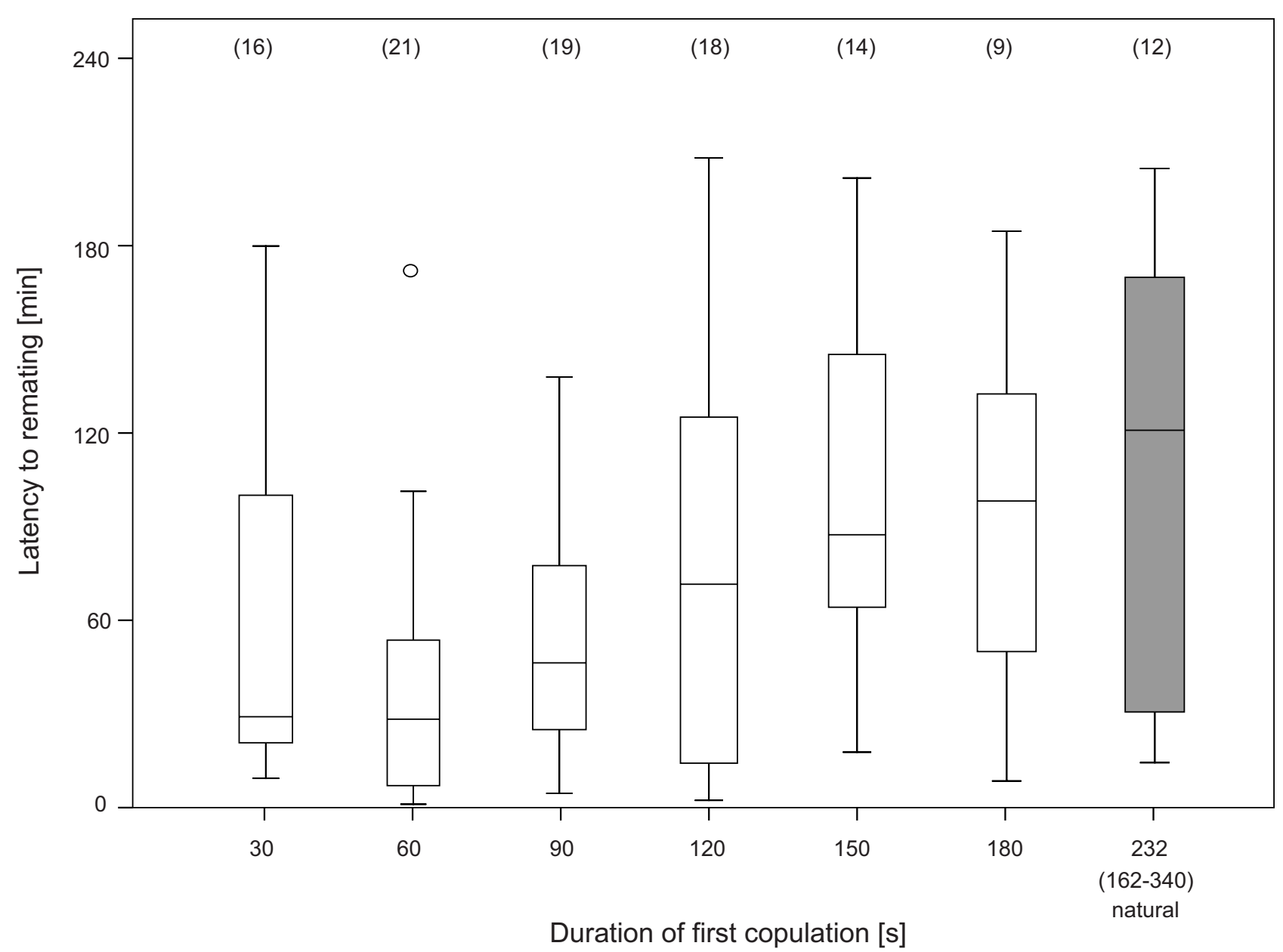

\section{Figure 5}

Latency to remating depending on duration of first copulation. Latency to remating (in minutes from introduction of the second male until copulation) $24 \mathrm{~h}$ after first matings that were either experimentally interrupted (white boxes) or naturally terminated (shaded box). The box plots represent the median as the middle line, 25 and $75 \%$ quantiles as box boundaries, and 10 and $90 \%$ quantiles as whiskers. Sample sizes are given in parentheses.

oratory, females readily remate when given the opportunity, some within minutes, and ca $40 \%$ within one day ([41] and personal observations). As female cooperation is an essential prerequisite for copulation to occur in $D$. montana [54], remating is likely an adaptively favoured female strategy, rather than an inability to physically resist coercive manipulation. Whether the reported delay in remating associated with longer first copulations imposes a significant fitness penalty is an issue that merits further attention, as is the investigation of other potentially incurred costs in terms of lifetime fecundity and of survival.

\section{Conclusion}

While male interests in sexual and sexually antagonistic selection scenarios have been studied intensively and are rather straightforward, females' interests have only lately come under scrutiny. The fact that the general effect is notoriously difficult to gauge in females (see chapter 6 in [26]) may account for this delay in interest. Our study provides empirical evidence for substantial female control over copulation duration in a Drosophila species. We also show for the first time in a Drosophila virilis group species (a species that has lately been paid much attention because of its suitability for studying the genetic basis of co-evolving sexual behaviour) that the main cost to females and fitness benefit to males of prolonged copulations lay in the extended latency to female remating, leading to a conflict of interest between the sexes.

\section{Methods \\ General methods}

Flies used in experiments were collected from replicate culture plexiglas vials of three isofemale lines (strains) 


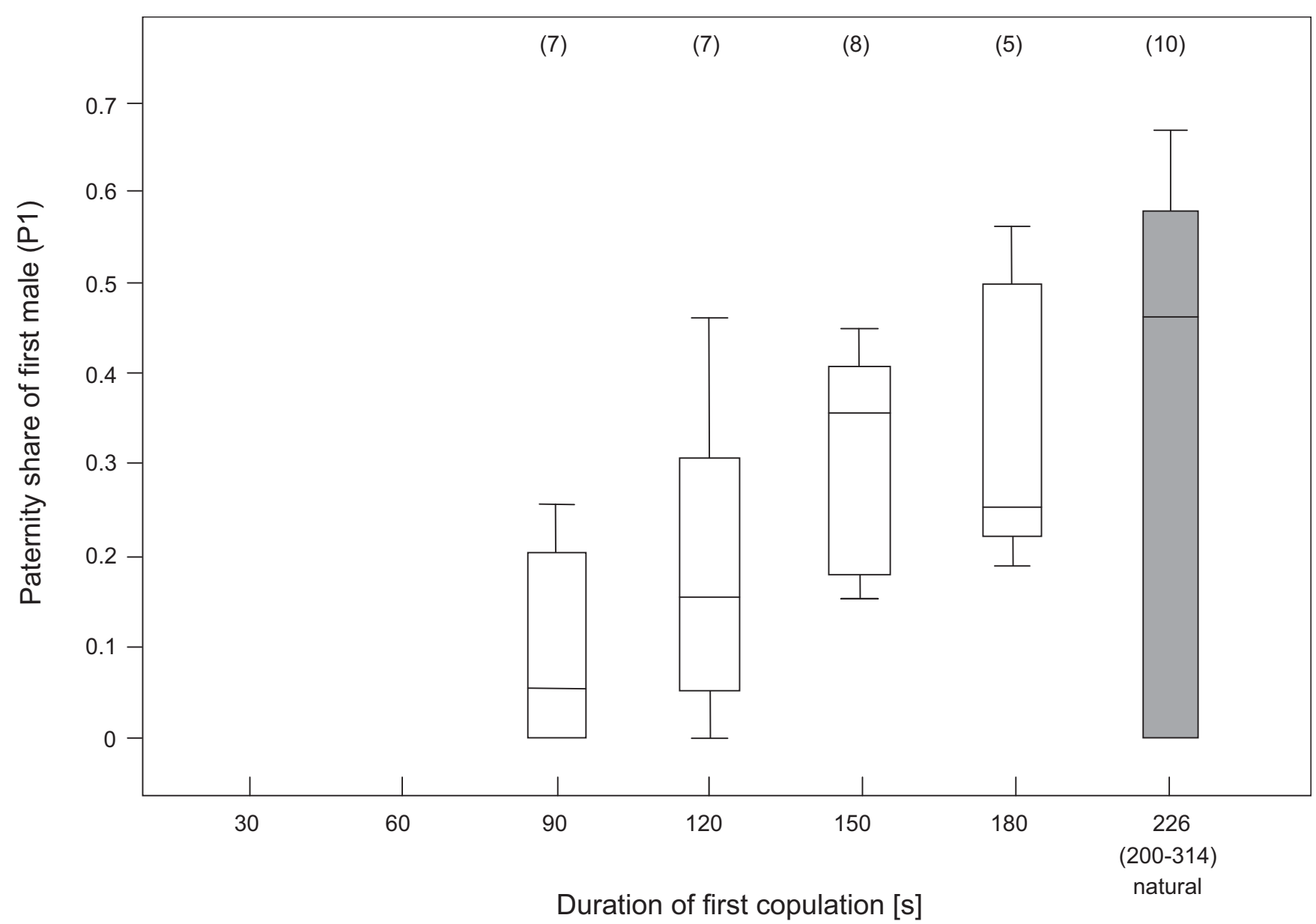

\section{Figure 6}

Proportion of progeny sired by the first male to mate depending on duration of first copulation. Proportion of progeny sired by the first male to mate $\left(P_{1}\right)$ when first matings were interrupted at predetermined times (white boxes) or allowed to be terminated naturally (shaded box) and second matings were allowed to be terminated naturally. The median (range) duration of copulation for unmanipulated first matings is given under the corresponding box. Sample sizes are given in parentheses.

from Yukon (Alaska, USA, $61^{\circ} 30^{\prime} \mathrm{N}, 159^{\circ} 20^{\prime} \mathrm{W}$ ), Oulanka (Finland, $66^{\circ} 25^{\prime} \mathrm{N}, 29^{\circ} 0^{\prime} \mathrm{E}$ ) and Kawasaki (Japan, $34^{\circ} 80^{\prime} \mathrm{N}, 139^{\circ} 60^{\prime} \mathrm{E}$ ) which had been inbred in the laboratory for over twenty generations (OSR and copulation duration experiments), and from isofemale lines established from collections made in 2003 in the surroundings of Vancouver (British Columbia, Canada, $49^{\circ} 16^{\prime} \mathrm{N}$, $122^{\circ} 55^{\prime} \mathrm{W}$, all other experiments). Experiments were conducted at the Universities of Jyväskylä, Finland (egg counts, hatching success, parentage analysis) and St Andrews, Scotland (OSR manipulation, larvae counts, sperm counts). The two laboratories have established identical methods, conditions and recipes, and have access to the same fly strains.

Strains were maintained at a density of approximately 50 flies per vial on malt medium sprinkled with live yeast and housed under constant conditions at a temperature of $19 \pm 0.5^{\circ} \mathrm{C}$ with continuous light. Males and females were collected within three days of emergence to ensure virginity. The virgin flies were sorted into separate sexes under light $\mathrm{CO}_{2}$ anaesthesia and placed in vials in single sex batches of five or six. The day preceding their use in experiments, males and females were isolated into individual vials. All flies were used upon reaching sexual maturity (age $21 \pm 2 \mathrm{~d}$ ). Experimental matings were staged in food vials (height: $8 \mathrm{~cm}$, diameter $3 \mathrm{~cm}$ ) stood upright and held at room temperature $\left(18 \pm 1^{\circ} \mathrm{C}\right)$ under continuous lighting provided by light bulbs $(11 \mathrm{~W} / 827)$ suspended ca 40 $\mathrm{cm}$ above the vial rims.

\section{Sex ratio manipulation}

To examine whether males adjust the time spent in copula according to the perceived presence of potential competi- 
tors, we recorded the duration of matings under different operational sex ratio regimes. Virgin males and females were randomly allocated to one of four treatments, differing in the intensity of competition among males for access to females: (1) one female and one male, (2) three females and three males (3) one female and two males, and (4) one female and five males. The treatment consisting of groups of three females and three males was intended to partially control for the increased disturbance ensuing from higher densities expected in the treatment with the most skewed male-bias, while keeping an even sex ratio. However, typically, copulating couples were ignored by the other flies. Occasionally, one rival male would display close to the mating pair or try to mount the mating male. We never witnessed a successful take-over attempt. If anything, the other males would court the still available females (in the three males and three females treatment) or court each other (in the one female and five males treatment) in male-male chains, as described for some D. melanogaster mutants [55]. Females were placed into vials first, and then the appropriate number of males was added. Flies were handled without anaesthesia. We recorded the duration of copulation from when the genitalia first locked until the male dismounted. We noted the time of the onset of the female kicking behaviour and calculated the length of the struggle between partners. In the treatment involving groups of three females and three males, we considered the first copulation to occur. Only males and females from Finland and Japan were used. The four resulting strain combinations (male or female from Finland or Japan) were evenly distributed across treatments. Up to eight vials were watched simultaneously, although no more than three replicates per treatment ran concurrently at any one time. All females were used only once, regardless of whether they mated or not. Males from vials in which no copulation occurred within four hours were re-tested on the following day, in the same group and treatment. In between observations, such males were held individually in fresh food vials. Whether males were used for the first or the second time did not significantly affect their copulation duration $(P>0.5)$. Groups of males failing to obtain any copulation within two observation sessions on subsequent days were discarded. An insect pin stuck in the medium was added in the treatment with one single pair to provide the suitable control for the manipulation where female resistance was prevented (see section 'Control over copulation duration' below).

\section{Control over copulation duration}

In order to investigate which of the sexes holds control over the duration of copulation, we measured the length of copulations in which the females' attempts to dislodge their mates were precluded. Only males and females from the two strains Finland and Japan were used in this experiment, whereby the four strain combinations (male or female from Finland or Japan) were similarly represented. Females were killed by an overdose of $\mathrm{CO}_{2}$ and then carefully pinned through the thorax, slightly to the right of the midline (Karlsbader insect pins nr. 0, Ernst Wirkner, Germany). The pin was then stuck into the malt medium layering the bottom of a food vial. We used fine forceps to adjust the inclination of the body and the spreading of the wings in a fair imitation of the typical wing lifting posture with which $D$. montana females signal readiness to mate [56], and to which males invariably respond with mounting [57]. Occasionally, when the male vigorously courted the pinned female, showing an obvious willingness to mate, we removed the cotton wool plug sealing the vial and gently nudged the pinhead. Slight movement of the pinned dead female occasionally helped to persuade reluctant males to mount. Trials were watched continuously for up to four hours or until copulation occurred. Males that did not mate within four hours were reused up to two times on subsequent days. We recorded the duration of copulation (in seconds) from the time of intromission until the male withdrew its aedeagus and dismounted.

\section{Mating interruptions}

Experimental interruptions of matings at predetermined times were conducted to examine whether males that protract matings beyond the females' preferred duration are rewarded by increased progeny production. One virgin female and one virgin male were placed together in a food vial. Flies were handled without anaesthesia. We ensured that all vials contained similar amounts of malt medium. Vials were scanned continuously until copulation occurred. We noted copulation start as the time when the genitalia were first observed to lock. Pairs were randomly assigned to different copulation duration treatments. After either $30,60,90,120,150$ or 180 s, matings were interrupted by gently aspirating the pair up and down a thin rubber hose. Timings were accurate to within ten seconds. We had to forgo planned additional time intervals of $210 \mathrm{~s}$ and $240 \mathrm{~s}$, as most copulations were naturally terminated beforehand. Females that started resisting males ahead of their set time were omitted from all analyses. In an identically handled control group, matings were allowed to proceed undisturbed until naturally terminated. All males and females were used only once. Immediately after the end of copulation (forced or spontaneous), males were removed to prevent remating. Females whose productivity was to be assessed were left to oviposit in the vials for three days, after which they were moved to fresh vials to avoid excessive larval crowding. Once-mated D. montana females produce progeny for about six days [53], though most (fertilized) eggs are laid on the second to fourth day following insemination (own observation). The experiment was repeated three times, firstly to determine egg production and hatching success, 
secondly to determine offspring production by counting larvae and thirdly to estimate the amounts of transferred sperm per time unit.

\section{Egg production and egg hatching success}

Egg production and hatching success were determined for females that had been allowed to copulate for predetermined durations ( 14 to 29 females per time interval), and for 26 females that terminated copulations naturally. Eggs laid in the first six days following mating (into two vials per female) were counted under a binocular microscope. Eggs can easily be spotted as circular whitish dots on the surface of the medium. The sum of the number of eggs in the two vials of each female was used for analyses. Vials were then kept under the same conditions as described above for general fly maintenance. All adults emerging from those eggs were counted, and the proportion of hatched eggs was calculated (hatching success, i.e. survival to adulthood).

\section{Larvae production}

Larvae production was determined $12 \pm 1$ days following matings of predetermined duration for 14 to 20 females per time interval, and for 18 control females allowed to terminate copulation naturally. The upper layer of the medium, which contained all of the (second- and thirdinstar) larvae, was dissolved in warm water and dispersed on filter paper. Larvae were killed by drying the filter paper in a drying cupboard $\left(\mathrm{ca} 50^{\circ} \mathrm{C}\right.$ ) and then counted under a binocular microscope.

\section{Sperm transfer}

As in the above assays no progeny were produced in copulations lasting less than $90 \mathrm{~s}$, the question arose of whether any sperm at all was transferred or rather whether sperm was transferred but not utilized by the females during the earlier stages of copulation. For assessment of sperm transfer, the earliest interruption was made after 60 s. Ten to eleven pairs were tested in each time interval plus twelve pairs that were allowed to end copulation naturally. Immediately after the copulation was either experimentally or naturally terminated, the females were dissected under a deep $\mathrm{CO}_{2}$ anaesthesia in a standard insect ringer solution (PBS buffer). Sperm in uterus, spermatheca and receptaculum seminis was scored as present or absent, as sperm migration is easily detected in nondyed tissues. This rather crude method was applied because methods to quantify the amount of transferred sperm failed, most likely due to sperm morphology: $D$. montana has very long spermatozoa of ca $3 \mathrm{~mm}$ (for comparison: male thorax length ca $1.5 \mathrm{~mm}[58,59])$, which could not be straightforwardly extracted from the reproductive organs of the females. Attempts to dye the sperm heads with DNA-specific fluorescent dyes within the female reproductive tract to count transferred sperm failed.

\section{Double matings}

We conducted a double-mating experiment to test whether males that coerce females into protracted matings derive an advantage in the competition for paternity against a later rival. Females were mated twice on consecutive days with different males. Only Canadian strains were used. Males were chosen from two strains fixed for different-sized alleles at the microsatellite locus Mon5 (described by [60]), hence allowing unambiguous assignment of paternity of all offspring within a clutch with the use of only one marker. Male strains were randomly assigned to mate first or second. First matings were interrupted after either $90,120,150$ or $180 \mathrm{~s}$, or were left to end naturally.

\section{Female remating}

Females were remated on the following day. Latency to remating was measured as the time interval between the time of introduction of the second male and the start of remating. Females that refused their suitors' advances for up to four hours were scored as unwilling to remate. Females that were not courted by their assigned second partner were omitted from analyses. All of the second matings were allowed to proceed until naturally terminated. Males were stored in $70 \%$ ethanol for molecular analyses. Females were given a 9-day egg laying period and were then discarded. Upon emergence, 16-20 offspring per female were collected for DNA extraction and paternity analyses.

\section{Paternity analyses}

Genomic DNA from single flies was isolated by the "single fly DNA preps" method [61]. The microsatellite locus Mon5 [60] was amplified in $10 \mu \mathrm{l}$ reactions containing $50-100 \mathrm{ng}$ of genomic DNA, $1 \mu \mathrm{M}$ of each primer (TAGC, Copenhagen, Denmark), $200 \mu \mathrm{M}$ dNTPs, $1.5 \mathrm{mM} \mathrm{MgCl}_{2}$ and 1 unit Taq DNA polymerase (Biotools B\&M Labs, Madrid, Spain) in $1 \times$ manufacturer's buffer. The reaction profile included denaturation at $94^{\circ} \mathrm{C}$ for $3 \mathrm{~min}$, then $94^{\circ} \mathrm{C}$ for $50 \mathrm{~s}, \mathrm{~T}_{\mathrm{a}}{ }^{\circ} \mathrm{C}$ for $50 \mathrm{~s}, 72^{\circ} \mathrm{C}$ for $50 \mathrm{~s}$ for 34 cycles, then a final extension at $72^{\circ} \mathrm{C}$ for $4.5 \mathrm{~min}$. $\mathrm{T}_{\mathrm{a}^{\prime}}$ the annealing temperature, was initially set to $4^{\circ} \mathrm{C}$ higher than $63^{\circ} \mathrm{C}$ as given in [60] and dropped by $1^{\circ} \mathrm{C}$ in each of the four initial cycles (from $63^{\circ} \mathrm{C}$ to $59^{\circ} \mathrm{C}$ ) to increase primer specificity. PCR amplification was performed on an MBS Satellite 0.2 G Thermal Cycler (Thermo Electron, Milford, MA, USA). The forward primers were fluorescently labelled for automated fragment sizing of PCR products run on an ABI Prism 3100 Sequencer (Applied Biosystems, Foster City, CA, USA) using GeneMapper v.3.7 (Applied Biosystems, Foster City, CA, USA). 


\section{Statistical analyses}

Data analysis was performed using JMP 7.0 for the Mac and SPSS 15.0 for Windows. Normality and homogeneity of variances of all raw data and residuals from models were checked by Shapiro-Wilk and Bartlett's tests, respectively.

\section{Sex ratio manipulation}

Copulation durations (square root transformed to meet parametric assumptions) were compared using an ANOVA model with the fixed factor treatment (sex ratio manipulation). Optimality models $[17,62]$ provided an explicit ordered prediction of the effect of our sex ratio manipulation on the duration of copulation, thus justifying the examination of statistical tests for ordered alternative hypotheses, i.e. ordered heterogeneity (OH)-test [63] in addition to conventional unordered statistics. The $\mathrm{OH}-$ test incorporates the Spearman rank correlation $\left(\mathrm{r}_{\mathrm{s}}\right)$ between the observed and the expected orders in the calculation of a new test statistics $\mathrm{P}_{\mathrm{c}} \mathrm{r}_{\mathrm{s}^{\prime}}$ where $\mathrm{P}_{\mathrm{c}}$ is the complement of the probability value from the conventional non-directional test $\left(\mathrm{P}_{\mathrm{C}}=1-\mathrm{P}\right)$. $\mathrm{P}_{{ }_{\mathrm{C}}} \mathrm{r}_{\mathrm{s}}$ combines the magnitude information in the sample extracted by $\mathrm{P}_{\mathrm{C}}$ (the heterogeneity component) with the independent information extracted by $r_{s}$ (the ordering component). It thereby allows not only a test against the null hypothesis of no differences between treatments, but, additionally, to refute the null hypothesis in the direction of an anticipated alternative hypothesis. Given corresponding probability values are read from a graph (Figure 1 in [63]), and hence approximate.

\section{Mating interruptions}

Because of many unproductive interrupted matings, distributions were often skewed such that transformations failed to normalize the data. Data are thus analyzed using Kruskal-Wallis analysis of variance (ANOVA) followed by Tukey-type multiple comparisons after Zar [64] calculated by hand and verified using SsS 1.1a (Rubisoft Software GmbH, Eichenau, Germany).

\section{Authors' contributions}

DM and KK conceived of the study, designed all experiments and performed some of them, conducted the statistical analyses and wrote the manuscript. JK performed some of the experiments and all molecular analyses, and participated in data analysis and in drafting the manuscript. AH participated in the design and coordination of the study, and discussed its interpretation and presentation. All authors read and approved the final manuscript.

\section{Acknowledgements}

We thank Mike Ritchie for providing facilities in St Andrews, and for competent advice on experimental design and helpful comments on the manuscript. Concerning issues of sperm extractions and counts, Rhonda Snook and Nina Wedell were valued sources of advice, unfortunately only unsat- isfactorily implemented. Three anonymous reviewers provided constructive comments on an earlier version of the manuscript. The work was funded by the European Union Research Training Network (Contract No.: HPRN-CT-2002-00266).

\section{References}

I. Andersson M: Sexual selection Princeton, NJ: Princeton University Press; 1994.

2. Pizzari T, Snook RR: Sexual conflict and sexual selection: Chasing away paradigm shifts. Evolution 2003, 57: I 223-I 236.

3. Parker GA: Sexual selection and sexual conflict. In Sexual selection and reproductive competition in insects Edited by: Blum MS, Blum NA. London: Academic Press; 1979:123-166.

4. Chapman T, Arnqvist G, Bangham J, Rowe L: Sexual conflict. Trends Ecol Evol 2003, 1 8:41-47.

5. Rice WR: Sexually antagonistic male adaptation triggered by experimental arrest of female evolution. Nature 1996, $38 \mathrm{I}: 232-234$.

6. Rice WR: Dangerous liaisons. Proc Natl Acad Sci USA 2000, 97:I2953-I2955.

7. Van Valen L: A new evolutionary law. Evol Theory 1973, I:I-30.

8. Bateman AJ: Intra-sexual selection in Drosophila. Heredity 1948, 2:349-368.

9. Parker GA: Sperm competition and its evolutionary consequences in the insects. Biol Rev 1970, 45:525-567.

10. Wedell N, Gage MJG, Parker GA: Sperm competition, male prudence and sperm-limited females. Trends Ecol Evol 2002, I 7:313-320.

II. Kvarnemo C, Ahnesjö I: The dynamics of operational sex ratios and competition for mates. Trends Ecol Evol 1996, I I:404-408.

12. Gage MJG: Associations between body size, mating pattern, testis size and sperm lengths across butterflies. Proc $R$ Soc Lond B 1994, 258:247-254.

13. Møller AP: Sperm competition, sperm depletion, paternal care, and relative testis size in birds. Am Nat 1991, I 37:882-906.

14. Parker GA, Ball MA, Stockley P, Gage MJG: Sperm competition games: a prospective analysis of risk assessment. Proc $R$ Soc Lond B 1997, 264: I793-I802.

15. Stockley $P$ : Sexual conflict resulting from adaptations to sperm competition. Trends Ecol Evol 1997, I 2: I54- 159.

16. Birkhead TR, Møller AP: Sperm competition and sexual selection London: Academic Press; 1998.

17. Parker GA, Ball MA, Stockley P, Gage MJG: Sperm competition games: Individual assessment of sperm competition intensity by group spawners. Proc R Soc Lond B 1996, 263: I 291-1297.

18. Hunter FM, Harcourt R, Wright M, Davis LS: Strategic allocation of ejaculates by male Adélie penguins. Proc $R$ Soc Lond B 2000 , 267: $1541-1545$.

19. Wedell N, Cook PA: Butterflies tailor their ejaculate in response to sperm competition risk and intensity. Proc $R$ Soc Lond B 1999, 266:1033-1039.

20. Schaus JM, Sakaluk SK: Ejaculate expenditures of male crickets in response to varying risk and intensity of sperm competition: not all species play games. Behav Ecol 200 I, I 2:740-745.

21. Sakaluk SK, Müller JK: Risk of sperm competition mediates copulation duration, but not paternity, of male burying beetles. J Insect Behav 2008, 2 1:153-163.

22. Alcock J: Postinsemination associations between males and females in insects: the mate-guarding hypothesis. Annu Rev Entomol 1994, 39:1-21.

23. Edvardsson M, Canal D: The effects of copulation duration in the bruchid beetle Callosobruchus maculatus. Behav Ecol 2006, I 7:430-434.

24. Duvoisin N, Baer B, Schmid-Hempel P: Sperm transfer and male competition in a bumblebee. Anim Behav 1999, 58:743-749.

25. Merritt DJ: The morphology of the phallosome and accessory gland material transfer during copulation in the blowfly, Lucilia cuprina (Insecta, Diptera). Zoomorphology 1989, 108:359-366.

26. Simmons LW: Sperm competition and its evolutionary consequences in the insects Princeton, NJ: Princeton University Press; $200 \mathrm{I}$.

27. MacBean IT, Parsons PA: Directional selection for duration of copulation in Drosophila melanogaster. Genetics 1967, 56:233-239. 
28. Parsons PA, Kaul D: Mating speed and duration of copulation in Drosophila pseudoobscura. Heredity 1966, 21:219-225.

29. Kaul D, Parsons PA: Genotypic control of mating speed and duration of copulation in Drosophila pseudoobscura. Heredity 1965, 20:381-392.

30. Krebs RA: Function and genetics of long versus short copulations in the cactophilic fruit fly, Drosophila mojavensis (Diptera, Drosophilidae). J Insect Behav 1991, 4:221-233.

31. Patty RA: Investigation of genetic factors influencing duration of copulation in 'eastern' and 'western' Drosophila athabasca. Anim Behav 1975, 23:344-348.

32. Hirai $Y$, Sasaki $\mathrm{H}$, Kimura MT: Copulation duration and its genetic control in Drosophila elegans. Zool Sci 1999, 16:21 I-2। 4.

33. Jagadeeshan S, Singh RS: A time-sequence functional analysis of mating behaviour and genital coupling in Drosophila : role of cryptic female choice and male sex-drive in the evolution of male genitalia. J evol Biol 2006, 19:1058-1070.

34. Arnqvist G, Rowe L: Correlated evolution of male and female morphologies in water striders. Evolution 2002, 56:936-947.

35. Singh BN, Singh SR: Female remating in Drosophila ananassae : shorter duration of copulation during second mating as compared to first mating. J Biosci 1999, 24:427-43।.

36. Chapman T: Seminal fluid-mediated fitness traits in Drosophila. Heredity 200I, 87:5II-52I.

37. Chapman RF, Espelie KE, Sword GA: Use of cuticular lipids in grasshopper taxonomy: A study of variation in Schistocerca shoshone (Thomas). Biochem Syst Ecol 1995, 23:383-398.

38. Hoikkala $A$, Klappert $K$, Mazzi $D$ : Factors affecting male song evolution in Drosophila montana. Curr Topics Develop Biol 2005 , 67:225-250.

39. Simmons LW, Kvarnemo C: Ejaculate expenditure by male bushcrickets decreases with sperm competition intensity. Proc R Soc Lond B 1997, 264: I203-1208.

40. Parker GA: Sperm competition games: raffles and roles. Proc $R$ Soc Lond B 1990, 242:120-126.

41. Aspi J: Incidence and adaptive significance of multiple mating in females of two boreal Drosophila virilis -group species. Ann Zool Fenn 1992, 29:147-159.

42. Vincent $A$, Ahnesjö I, Berglund A: Operational sex ratios and behavioural sex differences in a pipefish population. Behav Ecol Sociobiol 1994, 34:435-442.

43. Alonso-Pimentel H, Papaj DR: Operational sex ratio versus gender density as determinants of copulation duration in the walnut fly, Rhagoletis juglandis (Diptera: Tephritidae). Behav Ecol Sociobiol 1996, 39: 17|-180.

44. Clark SJ: The effects of operational sex ratio and food deprivation on copulation duration in the water strider (Gerris remigis Say). Behav Ecol Sociobiol 1988, 23:317-322.

45. Vepsäläinen K, Savolainen R: Operational sex ratios and mating conflict between the sexes in the water strider Gerris lacustris. Am Nat 1995, 146:869-880.

46. Spieth HT: Courtship behavior in Drosophila. Annu Rev Entomol 1974, 19:385-405

47. Aspi J, Lumme J, Hoikkala A, Heikkinen E: Reproductive ecology of the boreal riparian guild of Drosophila. Ecography 1993, 1 6:65-72.

48. Gilchrist AS, Partridge $L:$ Why it is difficult to model sperm displacement in Drosophila melanogaster : the relation between sperm transfer and copulation duration. Evolution 2000, 54:534-542.

49. Arnqvist $G$, Andrés JA: The effects of experimentally induced polyandry on female reproduction in a monandrous mating system. Ethology 2006, I I 2:748-756.

50. Arnqvist G, Nilsson T: The evolution of polyandry: multiple mating and female fitness in insects. Anim Behav 2000, 60:145-164.

51. Fox CW, Rauter CM: Bet-hedging and the evolution of multiple mating. Evol Ecol Res 2003, 5:273-286.

52. Lorch PD, Chao L: Selection for multiple mating in females due to mates that reduce female fitness. Behav Ecol 2003, I 4:679-686.

53. Aspi J, Lankinen P: Frequency of multiple insemination in a natural population of Drosophila montana. Hereditas 1992 I I7:169-177.
54. Liimatainen J, Hoikkala A, Aspi J, Welbergen P: Courtship in Drosophila montana : the effects of male auditory signals on the behaviour of flies. Anim Behav 1992, 43:35-48.

55. Hall JC: Courtship among males due to a male-sterile mutation in Drosophila melanogaster. Behav Genet 1978, 8: I 25- I4I.

56. Vuoristo M, Isoherranen E, Hoikkala A: Female wing spreading as acceptance signal in the Drosophila virilis group of species. J Insect Behav 1996, 9:505-516.

57. Liimatainen JO, Hoikkala A: Interactions of the males and females of three sympatric Drosophila virilis -group species, D. montana, D. littoralis, and D. lummei, (Diptera: Drosophilidae) in intra- and interspecific courtships in the wild and in the laboratory. J Insect Behav 1998, I I:399-4I7.

58. Pitnick S, Markow TA, Spicer GS: Evolution of multiple kinds of female sperm-storage organs in Drosophila. Evolution 1999, 53:1804-1822.

59. Pitnick S, Markow TA, Spicer GS: Delayed male maturity is a cost of producing large sperm in Drosophila. Proc Natl Acad Sci USA 1995, 92:10614-10618.

60. Orsini L, Schlötterer C: Isolation and characterization of microsatellites in Drosophila montana and their cross-species amplification in D. virilis. Molec Ecol Notes 2004, 4:4I2-4I4.

6I. Gloor GB, Preston CR, Johnson-Schlitz DM, Nassif NA, Phillis RW, Benz WK, Robertson HM, Engels WR: Type-I repressors of P-element mobility. Genetics 1993, 135:81-95.

62. Ball MA, Parker GA: Sperm competition games: inter- and intra-species results of a continuous external fertilization model. J theor Biol 1997, 186:459-466.

63. Rice WR, Gaines SD: Extending nondirectional heterogeneity tests to evaluate simply ordered alternative hypotheses. Proc Natl Acad Sci USA 1994, 91 :225-226.

64. Zar JH: Biostatistical analysis 4th edition. Upper Saddle River, NJ: Prentice-Hall; 1999.

Publish with BioMed Central and every scientist can read your work free of charge

"BioMed Central will be the most significant development for disseminating the results of biomedical research in our lifetime. "

Sir Paul Nurse, Cancer Research UK

Your research papers will be:

- available free of charge to the entire biomedical community

- peer reviewed and published immediately upon acceptance

- cited in PubMed and archived on PubMed Central

- yours - you keep the copyright

Submit your manuscript here:

http://www.biomedcentral.com/info/publishing_adv.asp
BioMedcentral 